\title{
LA ORGANIZACIÓN RETÓRICA DEL GÉNERO MANUAL DE ECONOMÍA: UN DISCURSO EN TRÁNSITO DISCIPLINAR *
}

\section{THE RHETORICAL ORGANIZATION OF THE TEXTBOOK GENRE OF ECONOMICS: A DISCOURSE OF A DISCIPLINE IN TRANSIT}

\author{
GIOVANNI PARODI \\ Pontificia Universidad Católica de Valparaíso. Valparaíso, Chile \\ gparodi@ucv.cl \\ ENRIQUE BOUDON \\ Pontificia Universidad Católica de Valparaíso. Valparaíso, Chile \\ eboudonv@gmail.com \\ CRISTÓBAL JULIO \\ Pontificia Universidad Católica de Valparaíso. Valparaíso, Chile \\ cristobal.julio.vergara@gmail.com
}

\section{RESUMEN}

En este artículo se aborda el estudio del discurso académico de la Economía, basado en parte del Corpus PUCV-UCSC-2013, recolectado en dos carreras universitarias de dos universidades chilenas. Desde este marco, un primer objetivo de esta investigación es describir la organización retórica funcional de un conjunto de 38 textos, correspondientes al género Manual. En segundo término, se establece la frecuencia de ocurrencia de las movidas y los pasos retóricos identificados. Los resultados generales indican que existe una tendencia a la homogeneidad en la organización discursiva de los treinta y ocho manuales, es decir, todos ellos ejecutan la mayoría de los pasos y movidas prototípicas. La cuantificación de la ocurrencia de algunos pasos retóricos reveló la existencia de algunas funciones presentes en todos los manuales (Plantear Ejercicios o Ejemplos), pero de otras que se realizan escasamente (Resolver la Tarea). Estos rasgos hacen que los manuales analizados, por una parte, se asemejen a los de Ciencias Básicas, pero - por otra- los llevan a acercarse a los de Ciencias Sociales y Humanidades. Esta tendencia dual o mixta en el discurso académico de la Economía refuerza el carácter controversial de un discurso que se hibridiza en el proceso de buscar la objetividad y cientificidad. Otro hallazgo lo constituye la identificación en algunos manuales de una suborganización incrustada en la Macromovida 2, a

\footnotetext{
* Proyecto FONDECYT 1130033.
} 
la que hemos denominado Nodo de Expansión y Reforzamiento Conceptual. Junto a ello, también destaca el análisis en términos multimodales, en donde emerge la diagramación como un componente relevante en la construcción de significados orientados por propósitos didácticos.

Palabras clave: Manual, discurso académico, Economía, diagramación multimodal, movidas retóricas.

\section{ABSTRACT}

In this paper we study the academic discourse of Economics, based in part of the PUCVUCSC-2013 Corpus, collected in two university careers of two Chilean universities. From this framework, the first objective of this research study is to describe the functional rhetorical organization of thirty-eight texts belonging to the Textbook genre. The second objective seeks to determine the frequency of occurrence of the moves and steps identified. The overall results indicate a tendency towards homogeneity in the discursive rhetorical organization of the textbooks under study. This means most of these texts execute most of the most prototypical moves and steps. The quantification of the occurrence of some rhetorical steps revealed the existence of certain features present in all textbooks (Presenting Exercises or Examples), but others perform poorly (Solving Task). These features make these textbooks, on the one hand, resemble the Basic Sciences texts, but -on the other- they make textbooks approach the Social Sciences and Humanities ones. This dual or mixed tendency in the academic discourse of Economics reinforces the controversial character of a discourse that hybridizes in the process of seeking objectivity and scientificity. Another finding is the identification in some textbooks of an embedded suborganization in Macromove 2, which has been called Node of Conceptual Expansion and Strengthening. Along with this, the analysis in multimodal terms also highlights another finding: the layout of some moves and steps emerge as an important component in the construction of meaning oriented by didactic purposes.

Keywords: Textbook, academic discourse, Economics, multimodal layout, rhetorical moves.

Recibido: 01.04.2014. Aprobado: 10.09.2014.

\section{INTRODUCCIÓN}

T as investigaciones acerca del discurso de la Economía han despertado interés $\_$por parte de economistas y lingüistas, fundamentalmente desde estudios basados en el inglés (McCloskey, 1983, 1985, 1994, 1999; Samuels, 1990; Bondi, 1995, 1996a, 1996b, 2002, 2010; Klamer, 1990; Henderson, Dudley-Evans \& Backhouse, 1993; Erreygers \& Jacobs, 2005; Bargiela-Chiappini, 2009; Maci \& 
Salas, 2012). Comparativamente, ha sido menor la indagación de la lengua española (Mateo, 2007; Martínez, 2009; Stagnaro, 2010, 2011, 2012; Moyano, 2012; Nevado, 2012) y, en Chile, son incipientes las investigaciones basadas en este tipo de discurso (Parodi y Burdiles, 2013; Parodi \& Bocca; 2013; Parodi, 2014a y 2014b; Boudon y Parodi, 2014).

El estudio y caracterización del discurso académico escrito de la Economía en la formación universitaria de pregrado permite construir un panorama de la formación inicial del futuro especialista en el área de la Economía y constituye una aproximación a los mecanismos discursivos que posibilitan tanto el acceso como la construcción de los conocimientos fundamentales de la disciplina. Diversos autores han abordado la relación entre lenguaje y Economía, pero sin lugar a dudas fue McCloskey $(1983,1985,1994)$ quien abrió un debate relevante, tanto para economistas como para lingüistas, acerca de la naturaleza y evolución del discurso de la Economía. McCloskey ha denominado "giro retórico" al cambio que acontece en el modo de construir los significados por escrito en esta disciplina, pasando de un estilo más literario o narrativo a uno más técnico y matemático.

Por otro lado, como se sabe, el estudio de la organización retórica funcional de un género fue originalmente desarrollado por Swales $(1981,1990)$ para describir funcionalmente un apartado o sección del Artículo de Investigación Científica. En términos generales, este tipo de análisis se orienta hacia la segmentación de un texto en unidades retórico-funcionales específicas y busca identificar micropropósitos comunicativos particulares, en torno al macropropósito comunicativo del género. Entre otros, un aporte relevante de este tipo de estudios es la explicitación de la organización subyacente que puede orientar la formación de los futuros miembros de una comunidad discursiva.

En este contexto, en la presente investigación se busca identificar y describir la organización retórica funcional del género Manual de Economía, a partir de un subcorpus de 38 textos (Corpus PUCV-UCSC-2013), todos ellos recolectados como parte del currículo de formación universitaria en carreras de pregrado de dos universidades chilenas. Junto a ello, también se busca determinar la frecuencia de ocurrencia de las movidas y pasos retóricos identificados en los textos en estudio. En otras palabras, en el presente estudio se indaga en el modo en que se presenta y enseña la Economía a estudiantes en formación universitaria, es decir, se busca conocer los recursos discursivos que permiten acceder a la información especializada escrita y que posibilitan -en este sentido- la construcción del conocimiento disciplinar.

Este artículo se organiza del siguiente modo: en la primera parte se establece el marco teórico de referencia para esta investigación y se comentan algunas cuestiones referidas al discurso de la Economía, al concepto de género del discurso y, más específicamente, al género Manual. Posteriormente, se describen los procedimientos metodológicos y el corpus y, a continuación, se entregan los resultados 
y se discuten los hallazgos. Se cierra el trabajo con conclusiones y proyecciones.

\section{MARCO DE REFERENCIA}

\subsection{El discurso de la Economía}

Tal como se sostenía anteriormente, diversos investigadores, entre ellos economistas y lingüistas, han puesto su atención en el discurso de la Economía. Tanto para describirlo como para poner de manifiesto el carácter controversial de un discurso que aparentemente transita desde su pertenencia a las Ciencias Sociales y Humanidades hacia su aspiración por ser parte de un discurso más cercano a las Ciencias Básicas. Al respecto, Klamer (1990: 129) sostiene que el discurso de la Economía "is elusive and frustrating to anyone who tries to enter its maze of questions, terms, diagrams and models"; sin embargo, en su opinión, estas dificultades no disuaden a los estudiantes a ingresar a este campo de estudio.

Como ya se ha señalado, fue McCloskey $(1983,1985)$ quien abrió el debate respecto del tipo de discurso al cual se adscribiría la Economía, y llamó así la atención sobre el denominado "giro retórico". Esta autora plantea que, para justificar metodológicamente sus trabajos, los economistas se apoyan en una perspectiva filosóficamente obsoleta que en realidad no se usa en la práctica científica (McCloskey, 1983). Esta perspectiva corresponde a lo que en Filosofía de la Ciencia y Epistemología se ha denominado tradicionalmente como Inductivismo, cuyo extremo se materializó en el Positivismo. Desde este marco, el "giro retórico" dice relación con los recursos textuales y discursivos que se ponen en práctica a partir de la segunda mitad del siglo pasado, al momento de escribir y comunicar el conocimiento que se elabora en Economía. Al respecto, Balak (2006) y Mosini (2011) coinciden en sus planteamientos acerca de la necesidad de estudiar el discurso de la Economía y desentrañar si los rasgos narrativos o persuasivos (denominados literarios) son los que prevalecen o se han sustituido por otros de índole formal y matemática, tales como fórmulas, gráficos y métodos estadísticos. El así llamado "giro retórico" está orientado por un supuesto modernismo en el uso del lenguaje (McCloskey, 1983), el cual permitiría argumentar posturas enfatizando las evidencias objetivas, las pruebas cuantificables y los análisis positivos.

En este mismo sentido, Royce (1999) plantea que los economistas más elocuentes, cuyos argumentos se han posicionado por encima del resto, son aquellos que usan recursos matemáticos en su retórica, en el sentido del uso de técnicas descriptivas derivadas del álgebra, la geometría, el cálculo, entre otras. McCloskey (1985), por su parte, sostiene que la Economía ha preferido orientarse por la Física newtoniana, en la forma de ecuaciones matemáticas y métodos estadísticos. Los economistas, según argumenta McCloskey $(1985,1994)$, han llegado a adquirir 
así un lenguaje más matemático-formal que literario.

Al respecto, Boudon y Parodi (2014) señalan, desde un análisis empírico multimodal, que los manuales de Economía presentan un discurso marcado por la importante presencia de fórmulas y gráficos. Complementariamente, Parodi (2014a) también ha demostrado la impronta del sistema simbólico matemático en el discurso académico de la Economía, a partir del estudio de cuatro géneros: Informe de Política Económica, Artículo de Investigación, Guía de Ejercicio y Manual. En este estudio se revela que fórmulas, gráficos y tablas tienden a ser los artefactos multisemióticos de mayor ocurrencia en estos géneros académicos especializados. No obstante a que se empleen estos recursos, que aparentemente aportan objetividad y, por ende, cientificidad, el discurso de la Economía, según Bondi (1999), se basa más en la persuasión y la probabilidad, que en la demostración y la certeza. Al respecto, McCloskey (1985) destaca el empleo de un conjunto de procedimientos retóricos que están lejos de aportar cientificidad al discurso de la Economía: por ejemplo, atención a la introspección, estudio de casos, empleo de metáforas, apelación a la autoridad basada en la tradición, entre otros. Entonces, esta caracterización controversial del discurso de la Economía y el debate en torno a su carácter científico ofrecen un espacio interesante para el estudio de la epistemología del discurso de la Economía desde la lingüística y el estudio de la organización retórico-funcional, como el que aquí se propone.

\subsubsection{Discurso académico de la Economía}

En términos estrictos, la noción discurso académico refiere al conjunto de géneros discursivos que se escriben y se leen en contextos de enseñanza-aprendizaje (escuelas, universidades, contextos educacionales en general). Preferentemente, es la variante escrita de la lengua el medio para producir, transmitir y fijar el conocimiento disciplinar en diversas áreas de estudio (Parodi, 2008a), aunque, sin lugar a dudas, el medio oral también juega un rol protagónico en los procesos de enseñanza, particularmente en aquellos géneros en que se produce interacción cara a cara, como por ejemplo, una cátedra dentro de una sala de clases. Estos géneros permiten acceder a la información especializada y cimentan la construcción de conocimientos disciplinares; de este modo, de manera gradual, integran a los estudiantes a una comunidad discursiva a través de un proceso conocido, en general, como alfabetización académica (Hall y López, 2011; Parodi, 2010a).

En este contexto, Oakey (2005) se interesa en el estudio de patrones fraseológicos y de los mecanismos evaluativos que se ponen en práctica en un género discursivo de la Economía, y lo hace a partir de un grupo de artículos de investigación en inglés. Por su parte, con un corpus de más de nueve millones de palabras, Goldschmidt \& Szmrecsanyi (2007) estudian también los artículos de investigación en Economía a partir de un importante número de revistas científicas en inglés. Entre 
otros, su objetivo es saber acerca de qué hablan los economistas. En una línea similar, Malavasi (2012) también estudia el discurso de la Economía en artículos de investigación, pero a través de un estudio contrastivo entre inglés e italiano. En lengua española, los estudios de Stagnaro $(2010,2011,2012)$ han abierto un espacio a la investigación, basada principalmente en artículos de investigación en revistas de Economía. Stagnaro ha explorado diversos aspectos lexicogramaticales que revelan, entre otros, qué analizan los economistas, cómo se presentan los objetivos y contenidos, y el modo en que se negocia el intercambio de información y se atenúa el compromiso de lo dicho.

Considerando estudios como los precedentes, establecer con precisión cuáles son los límites del discurso académico no es, ciertamente, una tarea simple, dado que se trata de prácticas comunicativas dinámicas, muchas de las cuales se llevan a cabo como parte de contextos sociales complejos (Flowerdew, 2002; Bolívar y Parodi, 2014). Así, buscando precisar las fronteras entre el discurso académico y el discurso profesional y aceptando que ellas son difusas, Bolívar y Parodi (2014) plantean que lo importante es estar conscientes de que la diferenciación principal está dada por el contexto de producción, el contexto de circulación, los participantes y los propósitos comunicativos. Por consiguiente, desde esta óptica, se entiende que el discurso académico comprende aquellos géneros discursivos, tanto orales como escritos, que posibilitan la construcción de significados en contextos de comunicación especializada entre estudiantes y profesores. De esta forma, si se usa un criterio estricto como ámbito o contexto de circulación, muchos géneros tradicionalmente considerados como profesionales podrían ser catalogados también como académicos, debido a que se encuentran circulando en una institución de educación y su rol, en ese contexto, sería pedagógico. Ello es lo que, por ejemplo, acontece cuando se identifica un Informe de Política Monetaria (IPoM) como parte de la bibliografía obligatoria de una asignatura de la línea de Economía en una carrera universitaria. Este género, elaborado por el Banco Central de Chile, proviene del ámbito profesional y no fue originalmente producido para un propósito educativo; sin embargo, en este contexto de uso, su función es netamente de enseñanza-aprendizaje. Tratando de especificar su naturaleza mixta y dinámica, a estos géneros como el IPoM, Parodi $(2012,2014 \mathrm{~b})$ los ha denominado géneros importados, ya que han sido trasladados a nuevos escenarios en una dimensión de conexión con el mundo profesional.

\subsection{Géneros del discurso}

En esta investigación compartimos el supuesto de que no existe una postura única

ni definitiva respecto de lo que se entiende por género del discurso y que, tal como 
indican -entre otros- Hyon (1996), Parodi (2008a) y Boudon y Parodi (2014), se reconocen diversas escuelas o aproximaciones, las cuales han tendido a enfatizar una dimensión por sobre otra. No obstante ello, en los últimos años el panorama parece estar cambiando y, como bien señalan Swales (2009), Russell, Lea, Parker, Street y Donahue (2009), Bazerman (2009) y Bhatia (2012), se detecta un avance hacia ciertos consensos.

Desde esta mirada integradora y multidimensional, entendemos que el género:

... constituye una constelación de potencialidades de convenciones discursivas, sustentada por los conocimientos previos de los hablantes/escritores y oyentes/lectores (almacenados en la memoria de cada sujeto), a partir de constricciones y parámetros contextuales, sociales y cognitivos. Dicho conocimiento -construido sociocognitivamente- se articula de modo operativo a través de representaciones mentales altamente dinámicas. Así, el género -como potencialidad de recursos- se instancia en conjuntos de selecciones convencionalizadas, las que presentan determinadas regularidades sincrónicamente identificables, pero que también son factibles de ser observadas a modo de variaciones diacrónicas, pues no son entidades de modo estático sino altamente dinámicas... (Parodi, 2008a: 26).

Así, buscando establecer precisiones y con el fin de contar con una definición operacional del constructo, en esta investigación adoptamos esta visión y adherimos a la naturaleza psico-socio-discursiva de los géneros.

\subsubsection{El género Manual}

El género Manual ha sido objeto de múltiples investigaciones en sus diversas manifestaciones a nivel escolar, universitario y profesional. Los diversos textos de este género ejercen un rol fundamental como pasajes de acceso a la construcción del conocimiento especializado en una variada gama de disciplinas y saberes (Kuhn, 1970; Swales, 1981, 1995; Klamer, 1990; Hyland, 1998; Kearsey \& Turner, 1999; Dimopoulos, Koulaidis \& Sklaveniti, 2005; Cubo de Severino, 2005; Parodi, 2008b, 2014a, 2014b; Martin, Maton \& Matruglio, 2010; Atienza \& Van Dijk, 2011; Oteíza y Pinto, 2011; Moyano, 2013; Boudon y Parodi, 2014). Desde esta perspectiva, el Manual se constituye como uno de los géneros prototípicos del discurso académico, pues -por una parte- su macropropósito comunicativo está íntimamente ligado con los procesos de enseñanza/aprendizaje y, por otra, sus núcleos retórico-organizacionales centrales tienden, más o menos regularmente, a la definición de conceptos y a la presentación de clasificaciones, ejemplificaciones y ejercicios (Parodi, 2008b). 
RLA. Revista de Lingüística Teórica y Aplicada, 52 (2), II Sem. 2014

En esta línea, Hyland (1998) establece que los manuales,

...play a central role in the learner's experience and understanding of a subject by providing a coherently ordered epistemological map of the disciplinary landscape and, through their textual practices, can help convey the norms, values and ideological assumptions of a particular academic culture (3).

Por su parte, Kuhn (1970: 135) define al Manual, en términos de su contenido y organización, como "cuerpo articulado de problemas, información y teorías". Cada área del conocimiento, sin embargo, posee características propias que las distinguen y, a partir de ello, Kuhn (1970) advierte el valor disímil que adopta el Manual a través de las diferentes disciplinas. De acuerdo a Kuhn (1970), en las Ciencias Sociales y Humanidades, el Manual posee un rol secundario, dado que, en esta área del conocimiento, los profesores exigen a sus estudiantes leer a los autores desde su fuente original, incluyendo desde la lectura de los clásicos de la disciplina hasta los artículos de investigación de estudios contemporáneos. Sin embargo, dentro del ámbito de las Ciencias Naturales ocurre todo lo contrario. El Manual encuentra un sitial preferencial dentro del proceso formativo de los estudiantes, puesto que ellos acceden al conocimiento especializado principalmente a través de estos textos que comprenden una parte fundamental de su formación (Kuhn, 1970; Parodi, 2008b, 2014b).

Esta distinción entre manuales, propuesta inicialmente por Kuhn (1970), tiene directa relación con la discusión acerca del estatus de la Economía como disciplina científica. Klamer (1990), en este sentido, sostiene que los manuales de Economía que circulan en el mercado sugieren que la disciplina posee un cuerpo de conocimiento bien desarrollado y articulado: "Indeed, economics texts convey the impression of consensus among economists, of a discipline that meets the standards of a hard science" (131).

Asimismo, podemos encontrar otros investigadores interesados en estudiar cómo se presenta la Economía a los estudiantes universitarios (Dudley-Evans \& Henderson, 1990; Hewings, 1990; Swales, 1993; Brown, 1993; Bondi, 1995, 1996a, 1996b). Henderson (1982), por ejemplo, ha indagado en el uso de metáforas y en cómo los economistas a menudo basan sus razonamientos en ejemplos hipotéticos y especulativos y no en lo que se presenta como hechos objetivos. Bondi (1995, 1996a, 1996b), entre otros temas, ha explorado el rol del lenguaje matemático, en cuanto modo de representación simbólica del razonamiento lógico y su función tanto referencial como explicativa a hechos que ocurrieron $\mathrm{u}$ ocurren en el mundo real o en alusión a mundos hipotéticos.

Ahora bien, siguiendo a Parodi, Venegas, Ibáñez y Gutiérrez (2008), optamos por una definición de este género que se orienta, principalmente, por el propósito comunicativo que cumple en el ámbito de la formación académica universitaria, 
la organización discursiva que presenta y los participantes involucrados. Así, un Manual se entiende como:

Género discursivo cuyo macropropósito comunicativo es instruir acerca de conceptos y/o procedimiento en una temática especializada. Su contexto de circulación ideal es el ámbito pedagógico y la relación entre los participantes es entre escritor experto y lector semilego o lego. Preferentemente, se hace uso de un modo de organización discursiva descriptivo y de recursos multimodales (Parodi et al., 2008: 60).

\section{MARCO METODOLÓGICO}

\subsection{Proyecto en marcha: construcción del Corpus PUCV-UCSC-2013}

En Chile, la formación universitaria en Economía se lleva a cabo por medio de -al menos- dos carreras de pregrado, a saber, Ingeniería Comercial y Contador Auditor. Dado que ambas carreras ofrecen una educación más integral y no exclusivamente centrada en Economía, se estudió cada plan de estudios y, en un trabajo estrecho con las autoridades y especialistas disciplinares de cada carrera, se identificó las asignaturas que se inscriben en la línea de la Economía. A partir de los programas de estas asignaturas, se revisó -en un primer momento- los materiales escritos allí consignados como lectura obligatoria o complementaria, pues se partió del supuesto de que en ellos está uno de los puntos de acceso a la construcción del conocimiento especializado.

De modo más específico, en la siguiente tabla se resumen los pasos llevados a cabo por nuestro equipo de investigación para construir el Corpus PUCVUCSC-2013 del discurso académico de la Economía.

Tabla I. Pasos metodológicos para la construcción del Corpus PUCV-UCSC-2013.

Paso 1: Construcción de una base de datos con la información acerca de las carreras que imparten el área de Economía en dos universidades chilenas

Paso 2: Contacto con los directores de las unidades académicas seleccionadas con el fin de solicitar los planes de estudio de las carreras y de los programas de todas las asignaturas de naturaleza obligatoria

Paso 3: Construcción de una base de datos con la información completa de los planes de estudio de las dos carreras en las dos universidades, así como de los respectivos programas de cada asignatura obligatoria 
Continuación Tabla I.

Paso 4: Contacto con directores de unidades académicas y con especialistas disciplinares en Economía con el fin de identificar la línea de formación en Economía en el plan de estudios y las asignaturas específicas

Paso 5: Construcción de una base de datos con todas las referencias bibliográficas incluidas en dichos programas de asignaturas del área de Economía

Paso 6: Contacto con los profesores de cada asignatura del área de Economía, con el fin de solicitar copia de materiales complementarios de lectura no incluidos como parte de las referencias en los programas oficiales (ya sea en formato papel o digital)

Paso 7: Búsqueda en Internet con el fin de encontrar aquellos títulos disponibles en formato digital, minimizando así el tiempo de digitalización

Paso 8: Recolección de los textos no encontrados en formato digital desde las bibliotecas correspondientes y de las oficinas de los profesores

Paso 9: Procesamiento y conversión de todos los textos del corpus en formato PDF editable y en formato plano $\left({ }^{*}\right.$ txt.)

Paso 10: Carga de cada texto a través del programa El Grial (www.elgrial.cl) en la plataforma en línea junto con su correspondiente procesamiento de etiquetado morfosintáctico

Una vez aplicados todos los pasos para la recolección del corpus en la primera fase, indicados en la Tabla II, los textos provenientes de todas las asignaturas de naturaleza obligatoria alcanzaron un total de 56. El corpus total, considerando también todos los materiales consignados como lecturas complementarias, se conformó con 222 textos.

\subsection{El subcorpus del género Manual}

Una vez construido el corpus y cerrado el proceso de recolección y digitalización de todos los materiales escritos, nuestro equipo se abocó a la identificación de los géneros del discurso. Para cumplir este objetivo se siguió los procedimientos indicados en Parodi, Venegas, Ibáñez y Gutiérrez (2008). De este modo, se identificó un conjunto de 38 textos pertenecientes al género Manual en el ámbito de la Economía.

Este género, dentro del Corpus PUCV-UCSC-2013 (Proyecto FONDECYT 1130033), ocupa un lugar preponderante en términos cuantitativos, dado que, tomando en consideración la bibliografía obligatoria declarada en los programas 
de las asignaturas, su frecuencia alcanza un $68 \%$ y su presencia y cantidad se constituye en un primer hallazgo que, preliminarmente, ofrece una importante caracterización del discurso académico de la Economía en dos instituciones de educación superior en Chile. Tal como señala Parodi (2014a), si la Economía se encuentra entre las disciplinas de las Ciencias Sociales y Humanidades, esta importante presencia de manuales en la formación universitaria contrastaría con el discurso académico de otras disciplinas del área, tales como Psicología y Trabajo Social (Parodi, 2008c) e Historia, Literatura y Lingüística (Parodi, 2012, 2014b), en donde este género es de escasa o nula ocurrencia. Según este dato, el discurso académico de la Economía estaría más cerca de los discursos de la Ingeniería en Construcción y de la Química Industrial (Parodi, 2008b, 2008c) y de la Física, la Química y la Biotecnología (Parodi, 2012, 2014b).

\subsection{Procedimientos de análisis de los manuales}

Con el fin de identificar la organización retórica funcional en términos de pasos y movidas de los 38 textos pertenecientes al género Manual, se siguió el protocolo establecido por Parodi (2008b), basado en la propuesta original de Swales (1990, 2004), complementado con otros desarrollos como los de Askehave y Swales (2001) y de Biber, Connor y Upton (2007). En la siguiente tabla, tomada de Parodi (2008b), se resumen los principales pasos metodológicos llevados a cabo para el estudio de las movidas en los manuales del corpus.

Tabla II. Descripción de las etapas y fases del análisis.

\begin{tabular}{|l|l|}
\hline $\begin{array}{l}\text { ETAPA 1: Configuración } \\
\text { del marco de análisis }\end{array}$ & $\begin{array}{l}\text { Se realiza un análisis preliminar a partir de un micro- } \\
\text { corpus para la construcción de una primera tabla de } \\
\text { criterios }\end{array}$ \\
\hline $\begin{array}{l}\text { Fase 1.1: Identificación de } \\
\text { unidades Discursivas }\end{array}$ & $\begin{array}{l}\text { A partir de una lectura analítica inicial, se identifica un } \\
\text { conjunto de unidades discursivas }\end{array}$ \\
\hline $\begin{array}{l}\text { Fase 1.2: Determinación del } \\
\text { foco de observación }\end{array}$ & $\begin{array}{l}\text { Se establece el grado de abstracción para la observa- } \\
\text { ción de los propósitos comunicativos constituyentes } \\
\text { del género }\end{array}$ \\
\hline $\begin{array}{l}\text { Fase 1.3: Primera } \\
\text { ecualización }\end{array}$ & $\begin{array}{l}\text { Revisión y ajuste de la relación entre el foco de obser- } \\
\text { vación y las unidades discursivas identificadas }\end{array}$ \\
\hline $\begin{array}{l}\text { Fase 1.4: Asignación de } \\
\text { propósitos comunicativos }\end{array}$ & $\begin{array}{l}\text { Se asocia cada unidad discursiva identificada a un pro- } \\
\text { pósito comunicativo }\end{array}$ \\
\hline $\begin{array}{l}\text { Fase 1.5: Producción de } \\
\text { etiquetas }\end{array}$ & $\begin{array}{l}\text { Se asigna una etiqueta a cada unidad discursiva iden- } \\
\text { tificada de acuerdo al propósito comunicativo que, } \\
\text { eventualmente, cumple }\end{array}$ \\
\hline
\end{tabular}


Continuación Tabla II.

\begin{tabular}{|c|c|}
\hline $\begin{array}{l}\text { Fase 1.6: Identificación del } \\
\text { macropropósito } \\
\text { comunicativo }\end{array}$ & $\begin{array}{l}\text { Se determina el macropropósito comunicativo del gé- } \\
\text { nero de acuerdo al conjunto de propósitos comunica- } \\
\text { tivos previamente identificados }\end{array}$ \\
\hline $\begin{array}{l}\text { Fase 1.7: Diseño de primera } \\
\text { tabla de criterios }\end{array}$ & $\begin{array}{l}\text { En base a las fases anteriormente desarrolladas, se di- } \\
\text { seña una primera grilla de clasificación en términos de } \\
\text { macromovidas, movidas y pasos }\end{array}$ \\
\hline $\begin{array}{l}\text { ETAPA 2: Extensión y } \\
\text { ajustes }\end{array}$ & $\begin{array}{l}\text { Se realiza la aplicación de la tabla de criterios al total } \\
\text { del corpus y se efectúan eventuales modificaciones }\end{array}$ \\
\hline $\begin{array}{l}\text { Fase 2.1: Aplicación de la } \\
\text { tabla de criterios }\end{array}$ & $\begin{array}{l}\text { Se aplica la tabla de criterios a la totalidad de los textos } \\
\text { del corpus }\end{array}$ \\
\hline $\begin{array}{l}\text { Fase 2.2: Segunda } \\
\text { ecualización }\end{array}$ & $\begin{array}{l}\text { A partir de la aplicación, se lleva a cabo las modifica- } \\
\text { ciones necesarias en la tabla de criterios. Ello implica la } \\
\text { inclusión o exclusión de algunas macromovidas, movi- } \\
\text { da y/o pasos }\end{array}$ \\
\hline $\begin{array}{l}\text { ETAPA 3: Validación de la } \\
\text { tabla de criterios }\end{array}$ & $\begin{array}{l}\text { Se realiza un proceso de triangulación para establecer } \\
\text { la confiabilidad del instrumento }\end{array}$ \\
\hline $\begin{array}{l}\text { Fase 3.1: Establecimiento } \\
\text { de la confiabilidad del } \\
\text { instrumento }\end{array}$ & $\begin{array}{l}\text { Se procede a la triangulación de la tabla de criterios } \\
\text { por parte de tres evaluadores expertos a partir de su } \\
\text { aplicación a un microcorpus }\end{array}$ \\
\hline $\begin{array}{l}\text { Fase 3.2: Tercera } \\
\text { ecualización }\end{array}$ & $\begin{array}{l}\text { A partir de la triangulación, se llevan a cabo ajustes } \\
\text { en la tabla de criterios para resolver las discrepancias } \\
\text { emergentes a partir del análisis de los pares expertos }\end{array}$ \\
\hline $\begin{array}{l}\text { ETAPA 4: Establecimiento } \\
\text { de la ocurrencia de las } \\
\text { categorías funcionales }\end{array}$ & $\begin{array}{l}\text { Se aplica el instrumento definitivo a la totalidad del } \\
\text { corpus con el fin de establecer la cuantificación de la } \\
\text { ocurrencia de las movidas y pasos }\end{array}$ \\
\hline Fase 4.1: Cuantificación & $\begin{array}{l}\text { Se cuantifica la ocurrencia de cada movida y paso en } \\
\text { cada texto constituyente del corpus }\end{array}$ \\
\hline
\end{tabular}

Junto a esta descripción del proceso y sus fases, en la presente investigación también se decidió aplicar el concepto y unidad de análisis denominado "Macromovida”, acuñado por Parodi (2008b), precisamente para el estudio de textos extensos como suelen ser los que pertenecen al género Manual. En el caso de los manuales de Economía, están constituidos por un promedio de 250.000 palabras, lo que hace que la unidad "Movida" no siempre logre dar cuenta cabalmente de funciones retóricas de mayor envergadura textual. Ahora bien, dado que este tipo de análisis se apoya en parte en lo estructural, pero busca ir más allá y estudiar unidades subyacentes en el plano del contenido y su función retórica, es relevante tener presente los planos de abstracción y poder observar unidades discursivas del tipo Macromovida (desde lo general a lo particular). No obstante ello, es fundamental considerar las pistas que determinadas organizaciones fraseológicas y 
léxicas aportan al reconocimiento de una unidad discursiva funcional. Como una forma de ilustrar más concretamente la descripción del procedimiento, el cual puede llegar a resultar un tanto abstracto, se seleccionó un pasaje de uno de los manuales en estudio. En el ejemplo presentado en la Figura 1, que es inicio de un capítulo, se muestran dos pasos específicos de la Macromovida 2 Conceptualización y Ejercitación, en su Movida Definición de Conceptos: Vincular Contenidos (VC) y Presentar Núcleo Temático (PN).

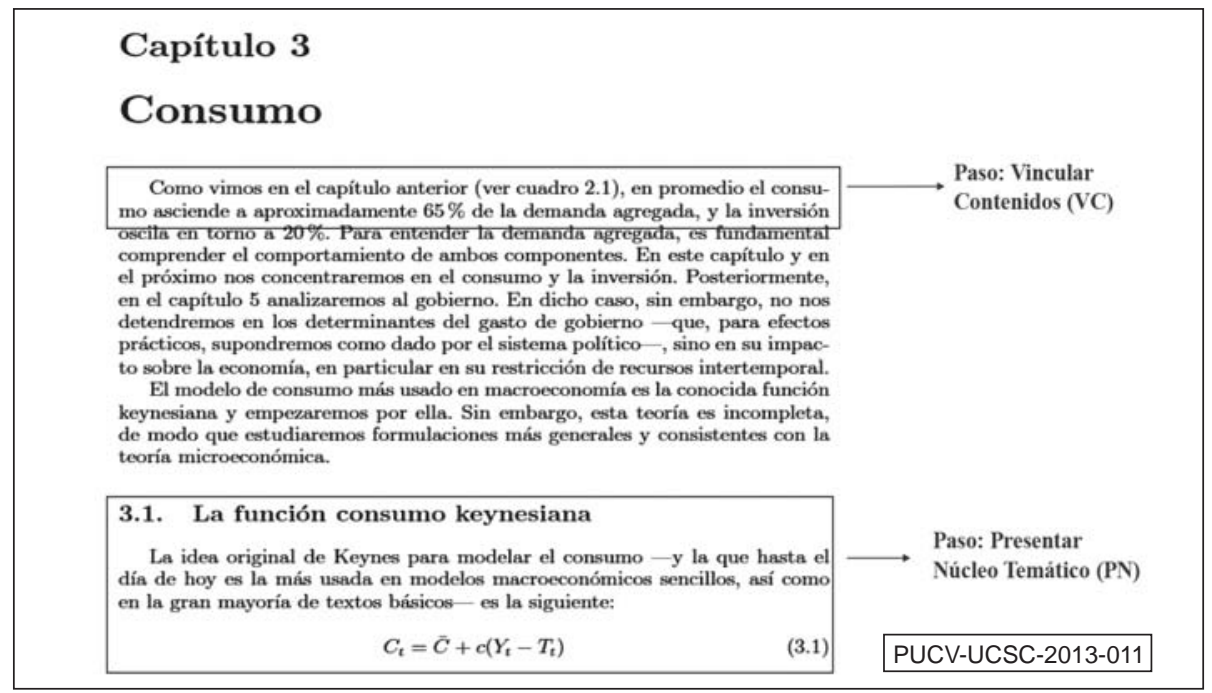

Figura 1. Movida Definición de Concepto.

Como se aprecia en el primer párrafo de este pasaje, el primer paso retórico (Vincular Contenidos: VC) está marcado lingüísticamente e introducido por la expresión "Como vimos en el capítulo anterior". El segundo paso (Presentar Núcleo Temático: PN), también en inicio de subapartado ya queda marcado por el subtítulo y luego se distingue por "La idea original de Keynes....", a lo que le sigue más adelante “...es la siguiente:...”. Vale la pena destacar el carácter multimodal de este paso en que parte del núcleo temático se presenta por medio de una fórmula.

\section{RESULTADOS: ANÁLISIS Y DISCUSIÓN}

\subsection{Organización retórica funcional}

La organización retórica funcional identificada en los 38 manuales analizados coincidió -de modo general- con los resultados encontrados en Parodi (2008b). 
Esto quiere decir que las macromovidas así como las movidas y sus respectivos pasos son los mismos que ya se habían detectado en manuales universitarios de otras disciplinas. A continuación, se presenta un esquema que resume las macromovidas, movidas y pasos identificados en esta investigación:

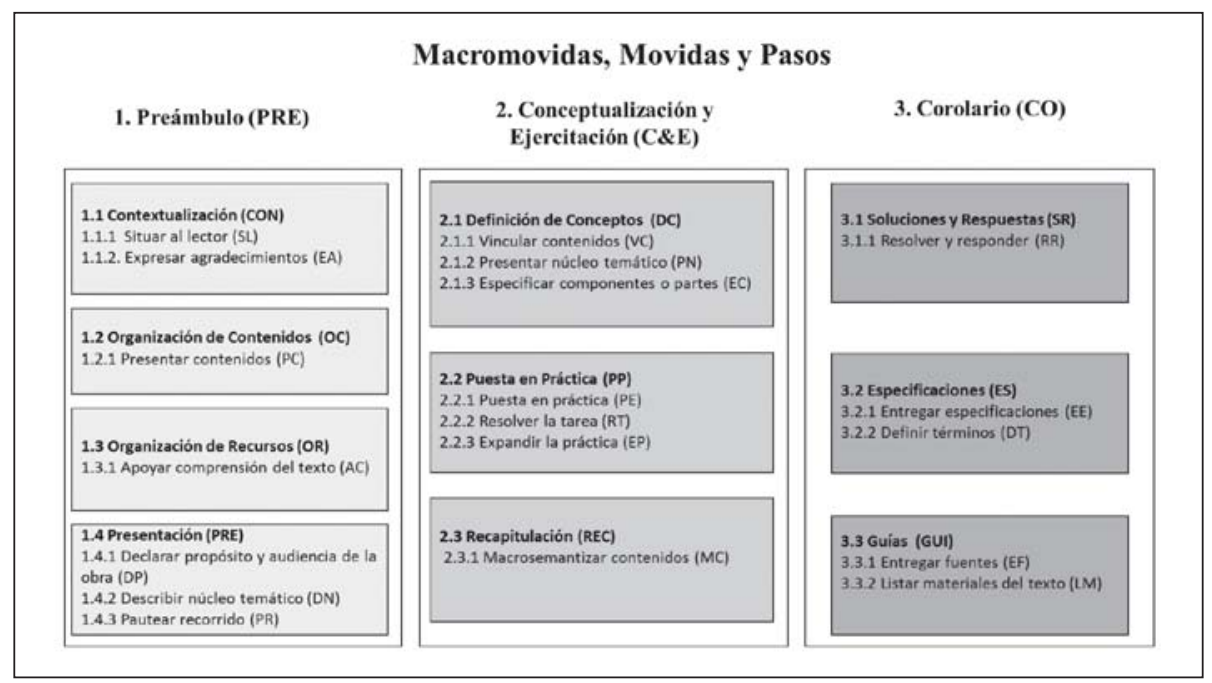

Figura 2. Macromovidas, Movidas y Pasos del Manual de Economía.

Como se puede apreciar, la organización del Manual de Economía revela una constitución retórica funcional que se despliega en tres macromovidas, diez movidas y diecinueve pasos. Esta descripción muestra claramente el carácter didáctico del género y su compromiso con la enseñanza/aprendizaje de contenidos específicos de la disciplina, lo cual pone de manifiesto una alta coherencia entre el macropropósito comunicativo del género y el despliegue de recursos conceptuales y didácticos. Desde la Macromovida 1 Preámbulo (PRE), se nota la especial atención a la audiencia, pues se le habla directamente y se le describe el propósito del texto y se le pautea el recorrido que se va a enfrentar. Seguidamente, la Macromovida 2 Conceptualización y Ejercitación (C\&E), núcleo del género, por una parte, pone en escena la definición de los conceptos a tratar, los describe y descompone y ejemplifica; y, por otra, lleva a cabo la ejercitación y la resolución de los problemas planteados. Por último, se retoman las ideas planteadas y se resumen los contenidos revisados. En la fase de cierre de la estructura funcional del género, la Macromovida 3 Corolario $(\mathrm{CO})$ ofrece solucionarios y expande la práctica con otros ejercicios y problemas; también se ofrecen glosarios y referencias bibliográficas.

Ahora bien, la descripción aquí presentada (de modo somero) también resulta 
relevante en su coincidencia con manuales de Psicología, Trabajo Social, Química Industrial e Ingeniería en Construcción (Parodi, 2008b, 2008c). Así, de modo general, el Manual presenta un plan retórico similar, solo alterado por algunas diferencias detectadas a través de las disciplinas en el componente ejercitación y resolución de problemas. Ellas dicen relación con un mayor énfasis en la enseñanza paso a paso de problemas y de resoluciones delineadas en el ámbito de la Ciencias Básicas (CB) (Parodi, 2008b, 2008c), hecho que se contrapone con la menor atención a la ejercitación y la escasa o nula entrega de solucionarios y pauteo de la resolución de ejercicios en los textos de las disciplinas de las Ciencias Sociales y Humanidades $(\mathrm{CS} \& \mathrm{H})$. Todo lo anterior revela los modos específicos de construir el conocimiento, de la epistemología disciplinar y de los rasgos distintivos prototípicos de unas y otras disciplinas y sus particulares comunidades discursivas (Swales, 1990, 2004; Hyland, 1998; Becher, 2001).

\subsection{Cuantificación de ocurrencias}

Un asunto relevante al momento de establecer los rasgos prototípicos de la organización retórica funcional de un género es determinar un índice estadístico que establezca las condiciones para considerar cuándo una movida o paso retórico tiene carácter obligatorio u optativo. Kanoksilapatham (2007), por ejemplo, propone que el $60 \%$ de ocurrencia es una medida potencial para establecer la estabilidad de una movida, por lo que sería el margen apropiado para determinar su obligatoriedad dentro de la organización retórica funcional de un género. Ibáñez (2008), para establecer qué movidas y pasos son obligatorios y opcionales en el estudio del género Texto Disciplinar, declara seguir este mismo principio metodológico. De igual modo lo hace Martínez (2012) en su estudio de tesis doctorales completas en dos disciplinas provenientes de dos países (Chile y España). Por su parte, Venegas (2014), guiado por el supuesto estadístico de la Distribución Lineal de la Información, determina el $70 \%$ de ocurrencia de una movida o paso como un margen aceptable para establecer la estabilidad estadística de una movida o paso retórico. En otras palabras, si la información se distribuye linealmente, por sobre el $70 \%$ de ocurrencia, no existiría variación estadística significativa dentro de un universo de datos.

Como criterio para esta investigación, hemos establecido el 60\% de ocurrencia como porcentaje indicador de que una movida es obligatoria dentro de la organización retórica funcional prototípica en los manuales de Economía, dado que, si bien existe cierta variación entre las decisiones entre los distintos investigadores, el rango estadístico divergente no resulta tan radical.

El Gráfico 1 despliega la distribución porcentual de las movidas en relación con su frecuencia de ocurrencia dentro del corpus en estudio. 


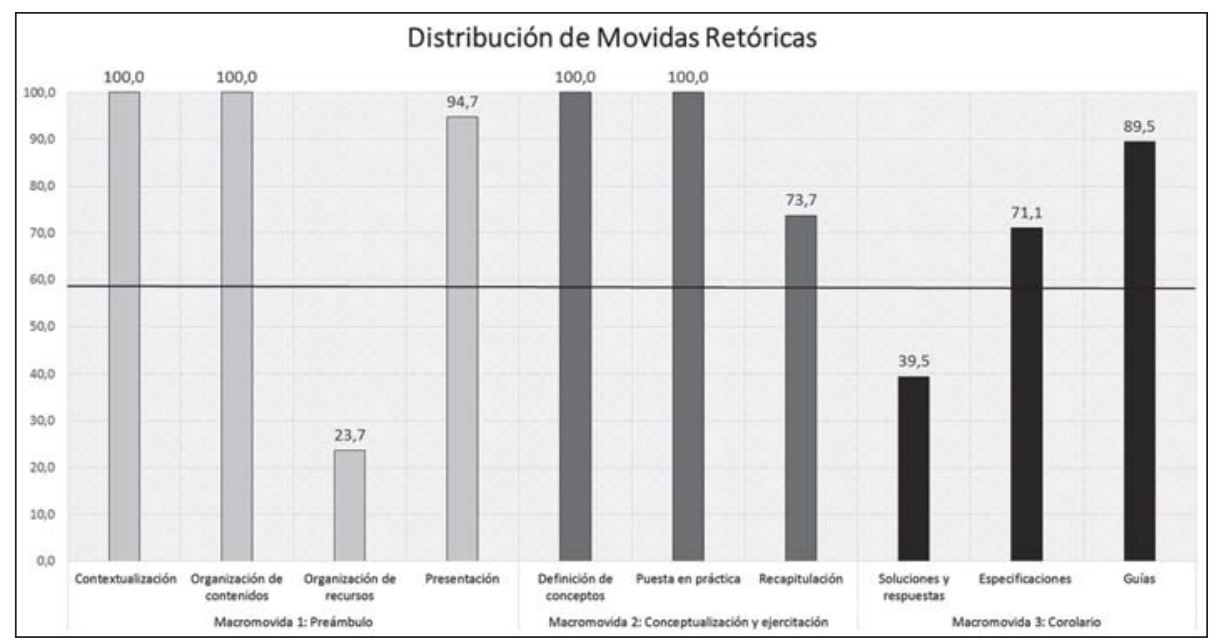

Gráfico 1. Ocurrencia de las movidas retóricas del género Manual en Economía.

La distribución de las cifras en el Gráfico 1 revela que la organización retórica funcional del Manual de Economía tiende a presentarse de manera relativamente homogénea, dado que la gran mayoría de las movidas identificadas en el apartado anterior despliegan una alta presencia en los ejemplares analizados (de las cuales un número importante supera el $70 \%$ en su frecuencia de ocurrencia). En concreto, ocho movidas alcanzan una frecuencia de ocurrencia superior al $60 \%$, dentro de las cuales destacan las movidas Contextualización (CON: 100\%), Organización de Contenidos (OC: 100\%), Definición de Conceptos (DC: 100\%) y Puesta en Práctica (PP: 100\%) con la ocurrencia máxima, seguidas de cerca por la movida Presentación (PRE: 94,7\%) y Guías (GUI: 89,5\%). Esto implica que el $80 \%$ de las movidas encontradas en la organización retórica funcional del Manual de Economía pueden ser catalogadas en el rango de obligatorias. Por el contrario, se desprende que, dada su baja frecuencia de ocurrencia dentro del corpus, las movidas Organización de Recursos (OR: 23,7\%) y Soluciones y Respuestas (SR: $39,5 \%$ ) despliegan un rol más secundario u opcional en la organización retórica funcional del Manual de Economía.

Estos hallazgos brindan datos interesantes acerca de la organización retórica funcional de los manuales de Economía. Desde el punto de vista del estatus disciplinar, la tendencia de estos manuales a presentar una alta frecuencia de ocurrencia en la mayor parte de las movidas de las tres macromovidas identificadas los acerca, por un lado, a los manuales de las Ciencias Básicas (CB) (Parodi, 2008c). No obstante, la baja frecuencia de ocurrencia de las movidas OR y SR establece un 
punto de encuentro con manuales provenientes del área de las Ciencias Sociales y Humanidades $(\mathrm{CS} \& \mathrm{H})$, puesto que estas movidas también presentan una escasa ocurrencia en manuales de Psicología y Trabajo Social (Parodi, 2008c). En este sentido, estos hallazgos brindan al discurso presente en los manuales de Economía un estatus mixto, en donde la organización retórica funcional muestra características prototípicas de manuales pertenecientes, por un lado, tanto al ámbito de las $\mathrm{CB}$, como - por otro- al de las CS\&H.

Con el objetivo de realizar un estudio más profundo de estos datos y, por consiguiente, ofrecer una descripción más detallada, a continuación se presenta la frecuencia de ocurrencia, esta vez, a nivel de los pasos identificados al interior de las movidas y macromovidas retóricas.

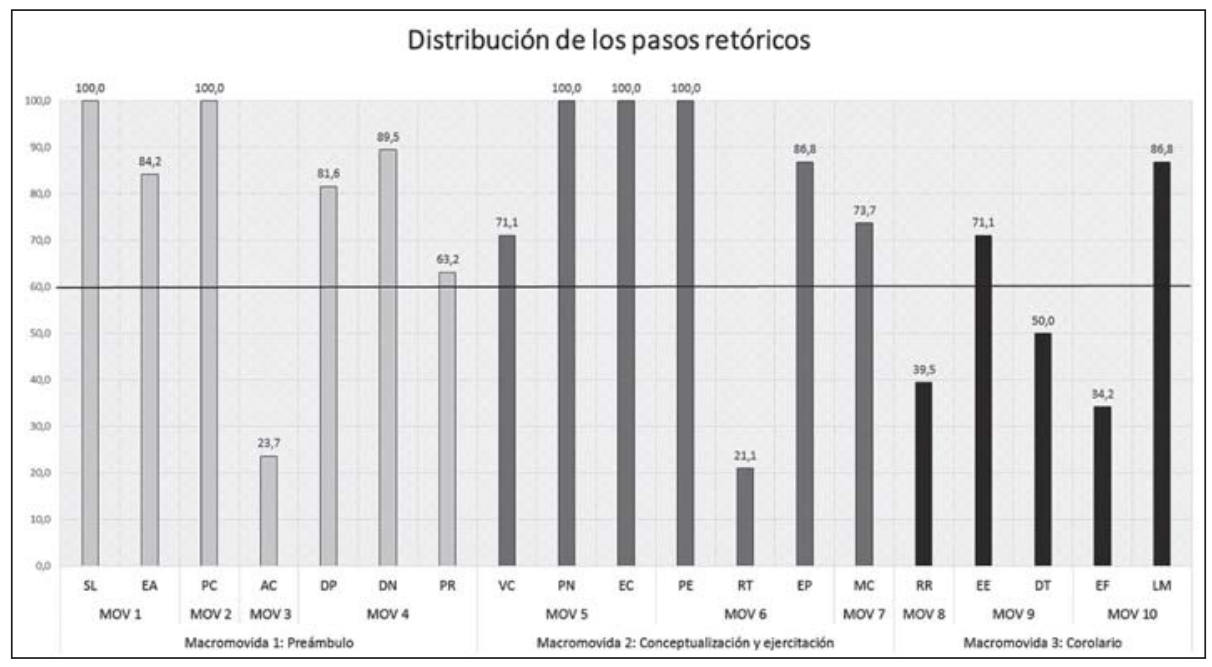

Gráfico 2. Ocurrencia de los pasos retóricos del género Manual en Economía.

Los datos expuestos en el Gráfico 2 ratifican la tendencia hacia una cierta homogeneidad en cuanto a la organización retórica funcional de este género, debido a que 14 -de un total de 19 - presentan una frecuencia de ocurrencia que supera el 60\%; junto a ello, cabe destacar que 13 de ellos muestran un rango superior al $70 \%$, esto es, el $68 \%$ de los pasos está por sobre el rango que se había establecido como umbral de corte para considerar como obligatorio un paso. Además, cabe destacar que 5 pasos alcanzan el 100\% de ocurrencia: Situar al Lector (SL), Presentar Contenidos (PC), Presentar Núcleo Temático (PN), Especificar Componentes o Partes (EC), y Plantear Ejercicios o Ejemplos (PE); estos pasos constituyen -en su conjunto- la parte central de un Manual, pues es allí donde se entregan 
los contenidos, se clasifican sus partes o componentes y se entregan problemas o ejemplificaciones acerca de lo que se está enseñando.

El caso más irregular lo exhibe la Macromovida 3, donde sólo dos de sus pasos constituyentes superan el 60\%, a saber, Entregar Especificaciones (EE: 71,1 \%) y Listar Materiales del texto (LM: 86,8\%), mientras que los pasos Resolver y Responder (RR: 39,5\%), Definir Términos (DT: 50\%) y Entregar Fuentes (EF: $34,2 \%)$ no superan el rango anteriormente señalado.

Estos hallazgos refuerzan la idea de la naturaleza mixta del discurso de los manuales de Economía. Por un lado, si ponemos nuestra atención en el componente práctico y didáctico, estos manuales se asemejan a los de las $\mathrm{CB}$, dado que otorgan un papel preponderante al planteamiento de problemas y a la ejercitación -Plantear Ejercicios o ejemplos (PE: 100\%) y Expandir la Práctica (EP: 86,8\%). No obstante lo anterior, los manuales de Economía estudiados presentan escasa tendencia a la entrega de un modelamiento paso a paso de cómo resolver los problemas y ejercicios planteados en el desarrollo de los contenidos (RT: $21,1 \%$ ) y a indicar explícitamente las respuestas a los ejercicios que cierran cada apartado (RR: $39,5 \%)$. Con el fin de ilustrar adecuadamente lo que se comenta, a continuación la Figura 3 muestra un ejemplo del paso RT en uno de los manuales analizados.

\begin{tabular}{|c|}
\hline Problema de demostración 1-1 \\
\hline $\begin{array}{l}\text { El director de Productos Automatizados está analizando la compra de una nueva } \\
\text { máquina que costará } 300.000 \text { dólares y tendrá una vida útil de cinco años. La máqui- } \\
\text { na permitirá reducir costes (al final del año) en Productos Automatizados, en una } \\
\text { cuantia de } 50.000 \text { dólares el primer año, } 60.000 \text { dólares el segundo, } 75.000 \text { el terce- } \\
\text { ro, y } 90.000 \text { dólares el cuarto y el quinto año. ¿Cuál es el valor actual de estos aho- } \\
\text { rros de costes generados por la máquina si el tipo de interés es del } 8 \text { por ciento? } \\
\text { ¿Debe el director comprar la máquina? }\end{array}$ \\
\hline $\begin{array}{l}\text { Respuesta: } \\
\text { Al gastar } 300.000 \text { dólares hoy en una nueva máquina, la empresa reducirá los costes } \\
\text { en } 365.000 \text { dólares a lo largo de cinco años. Sin embargo, el valor actual de los aho- } \\
\text { rros de costes es de tan sólo }\end{array}$ \\
\hline$V A=\frac{50.000}{1,08}+\frac{60.000}{1,08^{2}}+\frac{75.000}{1,08^{3}}+\frac{90.000}{1,08^{4}}+\frac{90.000}{1,08^{5}}=284,679 \$$ \\
\hline Por consiguiente, el valor actual neto de la nueva máquina es \\
\hline$C_{0}=284.679-300.000=-15.321$ dólares \\
\hline $\begin{array}{l}\text { Puesto que el valor actual neto de la máquina es negativo el director no deber } \\
\text { prarla. En otras palabras, el director podría ganar más invirtiendo } 300.000 \text { do }\end{array}$ \\
\hline
\end{tabular}

Figura 3. Ejemplo del paso Resolver la Tarea. 
Como es posible advertir en la Figura 3, el problema planteado es seguido de un modelamiento explícito de cómo debe ser solucionado. Así, se muestra paso a paso el razonamiento que se debe aplicar para resolver satisfactoriamente el problema en cuestión: si el director debe o no comprar la máquina. Para ello, el escritor se vale de diversos sistemas multisemióticos y de recursos de diagramación; por ejemplo, se emplea fórmulas y cifras matemáticas y se aprovechan las negritas y el tamaño de letra. En este contexto, la resolución que se alcanza, en el marco de las variables involucradas, parece ser la más acertada. No obstante ello, lo interesante de este ejercicio, desde el punto de vista lingüístico, se revela en el empleo de ciertos recursos que debilitan la conclusión; por ejemplo, la selección del modo condicional y el uso de verbos modales con que se entrega la respuesta: "el director no debería comprarla", "el director podría ganar más..." (en estos casos, las negritas son nuestras). El modo condicional del verbo abre la posibilidad y deja implícita la idea de que otra alternativa hipotética es "no invertir el dinero y sí comprar la máquina”. Al mismo tiempo, el empleo del verbo modal de obligación (deber) queda reducido en su fuerza ilocutiva de obligatoriedad al estar en modo condicional y la selección del verbo modal poder indica probabilidad y relativiza la ganancia del tipo de inversión. En otras palabras, a pesar del uso de recursos matemáticos y estadísticos en el razonamiento del problema, el modo de concluirlo involucra al lector y abre un mundo hipotético, alejado de los hechos concretos, en que se deja abierta otras posibles conclusiones al problema.

En síntesis, la organización retórica funcional de los manuales de Economía, por una parte, adopta características propias de discursos asociados a las CB, poniendo énfasis en ofrecer ejemplificaciones y proponer ejercicios y problemas a los contenidos disciplinares que se desarrollan. Pero, por otro lado, la manera de resolver los problemas propuestos, cuando esto sucede, sugiere, en algunos casos, una aproximación mixta en que se abre a mundos hipotéticos y se aleja de lo factual. Con ello, de cierto modo, el razonamiento en la resolución y conclusión suele ser más propio de los manuales de las CS\&H, los cuales ofrecen acercamientos alternativos a los fenómenos disciplinares o se suele dejar la respuesta abierta ante opciones competitivas según sea el caso particular (Kuhn, 1970; Parodi, 2008b); en definitiva, no existen leyes o reglas sino opciones alternativas en virtud de las variables involucradas y el juicio del lector.

\subsection{Variación en la Macromovida 2: Nodo de Expansión y Reforzamiento Conceptual}

Si bien, tal como ya se indicó, la organización retórica identificada en los manuales de Economía no revela ni más ni menos segmentos funcionales a los ya 
registrados previamente (Parodi, 2008b), sí se identificó variación en el modo de organización interna dentro de la Macromovida 2. Como ya había sido descrito por Parodi (2008b), los manuales están organizados en tres macromovidas, las que se despliegan en diez movidas. Mientras las macromovidas Preámbulo y Corolario no presentan un orden jerárquico en la organización de las movidas y pasos que las componen (existe intercambiabilidad), la Macromovida Conceptualización y Ejercitación sí presenta una secuencia progresiva que se repite canónicamente, coincidiendo -muchas veces- con la estructura en capítulos de los manuales. A ello, Parodi (2008b) denominó "colonia encadenada", tal como se presenta en la siguiente figura:

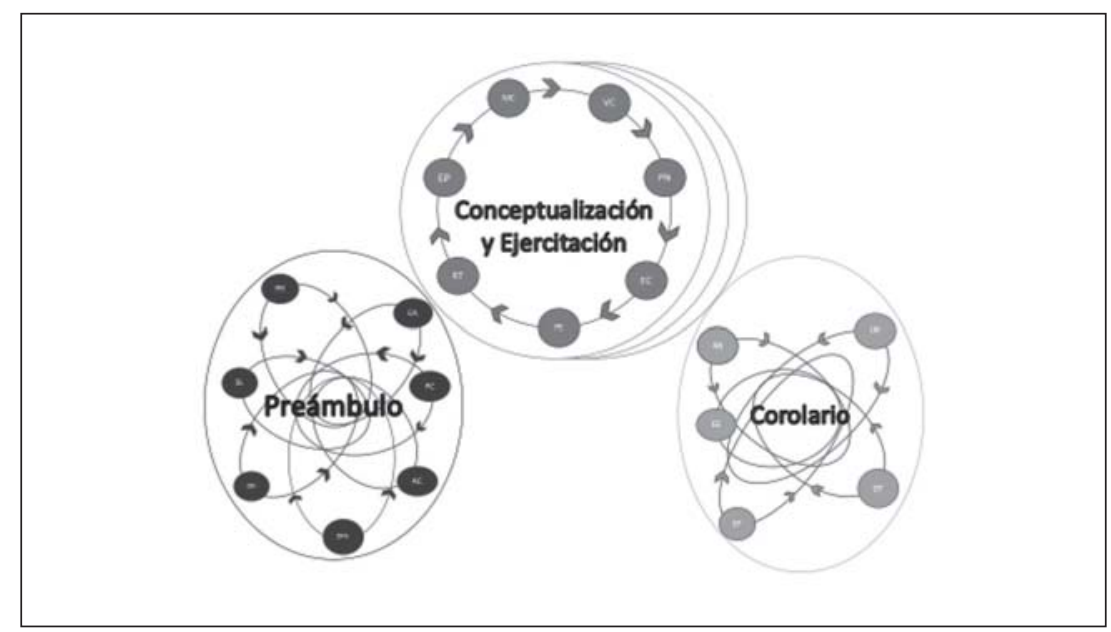

Figura 4. Posibilidades de ordenamiento de los pasos dentro de cada macromovida (Parodi, 2008b).

Esta colonia encadenada se compone de siete pasos retóricos, los que se presentan de forma estable a través de toda la Macromovida, la que, a su vez, también lo hace uniformemente a lo largo del Manual. En este sentido, en esa organización se detectan pasos retóricos que sólo ocurren una vez en la Macromovida 2, posicionándose en ciertos lugares de acuerdo a su propósito funcional. Por ejemplo, el paso Vincular Contenidos sólo se identifica al inicio de la Macromovida 2 y por una vez. No obstante ello, otros pasos, debido a la función que cumplen en la organización del género, sí pueden ocurrir nuevamente.

Desde este contexto, en los manuales de Economía se identificó una subconfiguración de pasos retóricos que se repite en la Macromovida 2, con énfasis en 
la expansión y reforzamiento conceptual. Esta configuración de pasos retóricos se acopla a los temas que se van tratando en el Manual con el propósito de reforzar la comprensión y asistir al estudiante en su proceso de lectura. De esta forma, la subconfiguración podrá ocurrir las veces que se presenten contenidos que el autor estime conveniente reforzar. Los pasos Especificar Contenidos (EC), Presentar Ejemplos y Ejercicios (PE), Resolver la Tarea (RT) y Macrosemantizar (MC) son los que componen esta subconfiguración, a la que hemos denominado Nodo de Expansión y Reforzamiento Conceptual.

Este modo de organizar los pasos retóricos -que devela una especial preocupación por el componente didáctico- está presente en el 61\% de los manuales en estudio. Con la intención de ilustrar de mejor manera el funcionamiento de estos nodos de expansión y reforzamiento conceptual, se presenta a continuación un rediseño de la Figura 4, que muestra la nueva organización de la Macromovida 2 Conceptualización y Ejercitación (Figura 5).

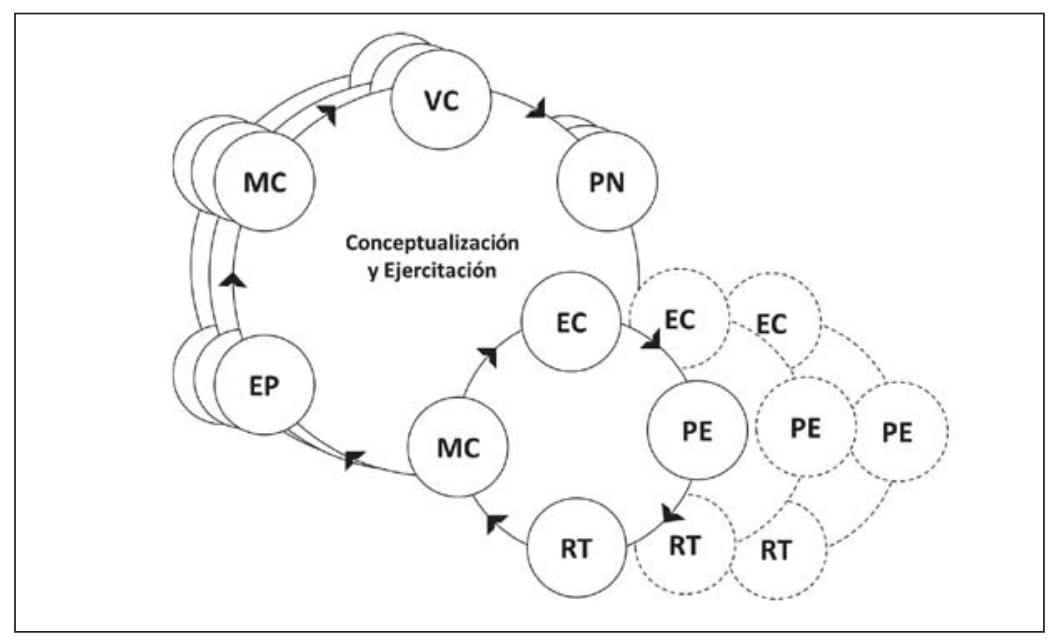

Figura 5. Nodo de Expansión y Reforzamiento Conceptual.

De acuerdo a la manera en que los manuales organizan los contenidos, la identificación de los nodos de expansión y reforzamiento conceptual permitió distinguir dos grupos, a saber, a) los manuales en donde la Macromovida 2 se organiza de forma idéntica a lo ya descrito por Parodi (2008b) y b) los manuales en donde la Macromovida 2 despliega una configuración particular, cuya organización agrupa pasos retóricos fundamentales desde el punto de vista didáctico. Según se ha podido comprobar, estos nodos se orientan a desarrollar distintos núcleos 
conceptuales, profundizando ciertas temáticas y, por consiguiente, robusteciendo el propósito comunicativo del género. Lo anterior deja en evidencia el mayor compromiso del autor con los procesos de enseñanza-aprendizaje, dado que se ha decidido enfatizar mecanismos que asistan la comprensión del estudiante/lector, junto con la puesta en práctica de los contenidos enseñados.

La identificación de estos nodos emergió, en parte, gracias al uso frecuente en estos textos de múltiples recursos multisemióticos; hecho que nos llevó a describir, exploratoriamente, la diagramación en este grupo de manuales.

\subsection{Diagramación en el Nodo de Expansión y Reforzamiento Conceptual}

Otro aspecto del estudio de la composición de los manuales dice relación con lo que se conoce como diagramación de la página o del texto. Según Kress (2010), la diagramación es identificada como un modo que posibilita la combinación de distintos sistemas, recursos y artefactos semióticos y, además, permite su distribución en términos de espacio. Entre estos recursos destacan los llamados encuadres, por medio de los cuales se resalta información relevante, delimitándola espacialmente y separándola de otros segmentos retóricos funcionales. También se identifica el empleo de la paleta de colores, que permite enfatizar un tipo de información de otra, asociada normalmente a un paso retórico particular (Van Leeuwen, 2011). Asimismo, el sistema tipográfico ocupa un lugar preponderante en la construcción de ciertos significados, mediante la utilización de diversos tamaños y tipos de letra, así como negritas y cursivas. Junto a lo anterior, también se detecta el uso de íconos de diversa naturaleza con los objetivos de marcar apartados, señalar ciertas funcionalidades, resaltar determinados pasos retóricos y llamar la atención del lector hacia contenidos más específicos o nucleares.

Considerando los 38 manuales, la diagramación con un alto componente multisemiótico se presentó en el $44 \%$ del total de los manuales en estudio. Como un modo de destacar la aparición de esta diagramación a que hacemos referencia, hemos seleccionado dos pasajes correspondientes a dos textos del corpus (Figura 6). 


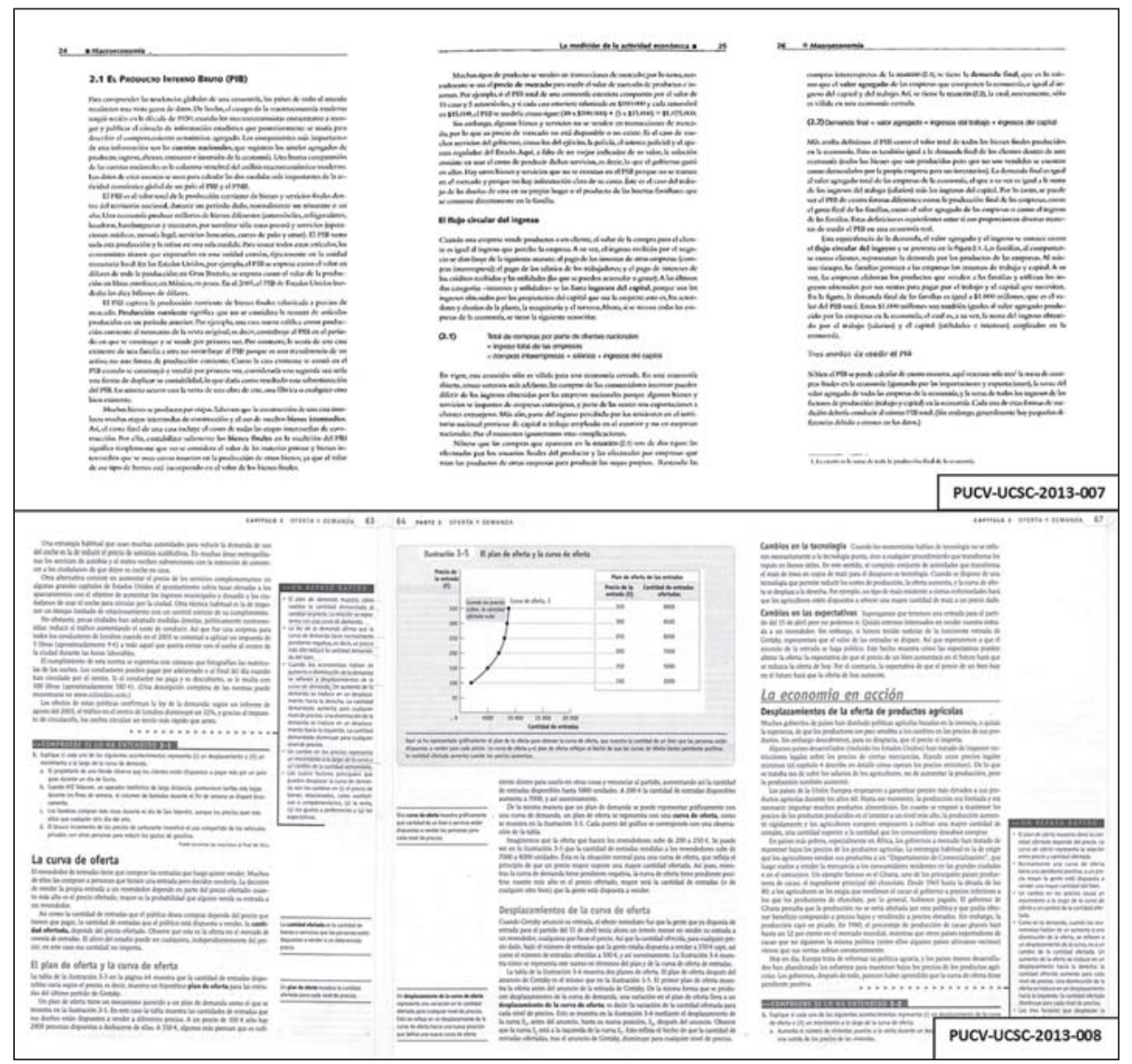

Figura 6. Comparación de la diagramación en dos manuales.

Según se puede apreciar en la silueta textual, existen abundantes diferencias en el modo de diagramar ambos manuales. El primer ejemplo (PUCV-UCSC-007) despliega sus contenidos con escaso uso de los recursos señalados previamente. En este sentido, sólo se nota el empleo del recurso tipográfico negrita al introducir los títulos y subtítulos, y de las viñetas para destacar la presentación de fórmulas. Por su parte, el segundo ejemplo (PUCV-UCSC-008) hace uso de una amplia gama de elementos de diagramación, además de los ya descritos para el ejemplo anterior. Así, es posible advertir la incorporación de distintos tipos de encuadre, a través de los cuales se resalta y delimita información de distinta naturaleza, tales como definiciones, ejemplificaciones, evaluaciones y resúmenes; también se emplea artefactos multisemióticos, como por ejemplo, un gráfico. Del mismo modo, 
se observa la utilización del tamaño y tipo de letra, los sombreados e íconos. Todo ello pone de manifiesto una configuración diagramática compleja, orientada a apoyar los procesos de enseñanza-aprendizaje. Cabe destacar que, junto al empleo de estos recursos, en estos mismos manuales también se identifica la subconfiguración singular de pasos retóricos; dicho de otro modo, existe una sinergia entre este modo de diagramación y la ocurrencia del Nodo de Expansión y Reforzamiento Conceptual, ya que ambos recursos se conjugan para dar forma al macropropósito comunicativo del Manual.

Junto a lo anterior, cabe señalar, además, que los manuales con mayor diagramación suelen presentar un número superior de instancias de indicaciones metatextuales, a través de las cuales se busca guiar y ayudar al lector a realizar ciertas tareas. También, en algunos casos, se estimula la reflexión metacognitiva mediante el uso de cierto tipo de indicaciones (por ejemplo: "Un repaso rápido", "Prueba rápida", "Compruebe si lo ha entendido"). A estas instancias textuales de interacción, Bondi (1999) las denomina diálogos entre autor y lector y constituyen, en opinión de la investigadora, una suerte de escenificación del diálogo en que -en muchos casos- el escritor se dirige directamente al lector y lo interpela a tomar conciencia de un contenido o a ejecutar una tarea determinada.

\section{CONCLUSIONES}

El análisis de la organización retórico-funcional de este grupo de 38 manuales de Economía, que corresponde al 100\% de los textos de este género discursivo en el Corpus PUCV-UCSC-2013, ha revelado que -en términos generales- su composición retórica funcional tiende a un alto grado de homogeneidad y la variación interna es escasa. De este modo, se ha identificado una organización genérica que en tres macromovidas, diez movidas y diecinueve pasos dan forma al macropropósito del género: "... instruir acerca de conceptos y/o procedimiento en una temática especializada” (Parodi et al., 2008: 60).

También se ha logrado determinar que la función retórica central del género se establece mediante la llamada Macromovida Conceptualización y Ejercitación, en la cual se presentan los núcleos temáticos seleccionados por el autor/escritor en virtud del tema general de la obra; del mismo modo, consecuentemente con el carácter didáctico del género, se ofrecen ejercicios y problemas y -en algún gradose desarrollan soluciones para ellos; también se pone especial énfasis en resumir y reiterar los conceptos abordados. Esta organización se asemeja a la identificada previamente por Parodi (2008b) en manuales de otras disciplinas de CS\&H y de CB.

Por otra parte, la cuantificación de las movidas y pasos permitió determinar que un número considerable de movidas y pasos ocurre en el 100\% de los manua- 
les y que otro número significativo presenta una frecuencia de ocurrencia sobre el 60 y 70\%, hallazgos que apoyan la conclusión de tendencia hacia una cierta homogeneidad de los textos estudiados. En este sentido, son escasas las movidas o pasos que muestran baja ocurrencia. Sólo sucede con cuatro de los diecinueve pasos: AC (Apoyar Comprensión del Texto), RT (Resolver la Tarea), RR (Resolver y Responder) y EF (Entregar Fuentes). De ellos, en nuestra opinión, lo más relevante es la baja ocurrencia de RT y de RR, pues estos pasos dicen relación con la resolución de los ejercicios y problemas planteados.

Este hallazgo pone de manifiesto el tránsito del discurso académico de la Economía entre una disciplina que, por un lado, se presenta cercana a la $\mathrm{CB}$ mediante el empleo de recursos matemático y estadístico y de, entre otros, gráficos y tablas (Parodi, 2010b; Boudon y Parodi, 2014; Parodi, 2014a) y ofrece ejercicios y problemas ante cada concepto enseñado; no obstante ello, en un importante número de manuales no se lleva a cabo la resolución de la tarea presentada, hecho más característico de los manuales de CS\&H (Parodi, 2008b, 2008c). Estos hallazgos son los que conducen a sugerir que el discurso académico de la Economía podría ser un discurso mixto o híbrido que busca posicionarse desde una perspectiva disciplinar cercana a las $\mathrm{CB}$, pero también se encuentra orientado por el discurso de las CS\&H.

Ahora bien, el estudio detallado de la Macromovida 2 Conceptualización y Ejercitación permitió identificar una cierta variación respecto de lo descrito por Parodi (2008b) en cuanto a la denominada Colonia Encadenada y su secuencia jerárquica de movidas y pasos. Esta nueva organización detectada, si bien mantiene el carácter cíclico y espiralado, ha mostrado -en un número importante de casos- resultar más flexible en su organización interna, revelando cierta reiteración de algunos pasos y una suborganización a través de algunas movidas a partir de básicamente cuatro pasos: EC (Especificar Componentes), PE (Plantear Ejercicios), RT (Resolver la Tarea) y MC (Macrosemantizar). A esta nueva suborganización incrustada se ha denominado Nodo de Expansión y Reforzamiento Conceptual, ya que a través de ella se busca profundizar en algunos conceptos y explorarlos más detalladamente. Al mismo tiempo, se ha identificado que los manuales que realizan el Nodo de Expansión y Reforzamiento Conceptual despliegan recursos multisemióticos de variada naturaleza para dar mayor énfasis a su papel didáctico y profundizar el contenido enseñado. Entre ellos, destaca el uso de la diagramación de la página en la que -por ejemplo- se realizan encuadres para destacar cierta información; además, el empleo de negritas, tamaños de letra, inserción de íconos y uso de la paleta de colores cumplen funciones relevantes en la construcción de los significados.

En síntesis, los hallazgos reportados en esta investigación llevan, por una parte, a detectar un cierto grado de hibridación del discurso de los manuales de Economía. Ello en cuanto se orquesta un conjunto de recursos retóricos que aportan 
objetividad al definir y ejemplificar conceptos, apoyados por el uso de artefactos multisemióticos de naturaleza matemática y estadística; pero, al mismo tiempo, los ejercicios planteados son escasamente resueltos paso a paso. Y, en ocasiones, cuando los manuales ejecutan este paso de resolución, tienden a usar expresiones que debilitan la asertividad de lo dicho, abriendo escenarios hipotéticos a las respuestas definitivas para el problema planteado. Por otra parte, este estudio ha avanzado más allá de los objetivos comprometidos y ha abierto una mirada del género Manual en su carácter multimodal, prestando especial atención a la diagramación para ejecutar pasos retóricos particulares.

Como parte de las proyecciones, cabe señalar la necesidad de llevar a cabo el estudio de los artefactos multisemióticos que constituyen parte de las movidas y pasos. Al respecto Boudon y Parodi (2014) y Parodi (2010b; 2014a) han identificado el tipo y la frecuencia de ocurrencia de, entre otros, fórmulas, gráficos y tablas en los textos de este género, por lo que resulta relevante conocer la configuración textual específica en que ellos se realizan. Del mismo modo, el tipo de relación intersemiótica a través de las que estos artefactos construyen significados en su interacción con el sistema verbal es importante, por lo que indagar en los mecanismos de cohesión y coherencia textual y los tipos de relaciones semánticas más recurrentes constituye otro polo de investigación futura.

\section{REFERENCIAS}

Askehave, I. \& Swales, J. (2001). Genre identification and communicative purpose: A problem and a possible solution. Applied Linguistics, 22(2), 195-212.

Atienza, E. \& Van Dijk, T. (2011) Knowledge and discourse in secondary school social science textbooks. Discourse Studies, 13(1), 93, 725-749.

Balak, B. (2006). McCloskey's rhetoric: Discourse ethics in Economics. London: Routledge.

Bargiela-Chiappini, F. (ed.). (2009). The handbook of business discourse. Edinburgo: Edinburgh University Press.

Bazerman, Ch. (2009). Genre and cognitive development: Beyond writing to learn. En Ch. Bazerman, A. Bonini \& D. Figueiredo (eds.), Genre in a changing world (pp. 279-294). Indiana: Parlor Press.

Becher, T. (2001). Tribus y territorios. La indagación intelectual y las culturas de las disciplinas. Barcelona: Gedisa.

Bhatia, V. (2012). Critical reflections on genre analysis. Ibérica, 24, 17-28.

Biber, D.; Connor, U. \& Upton, T. (2007). Discourse on the move. Using corpus analysis to describe discourse structure. Amsterdam: John Benjamins.

Bolívar, A. \& Parodi, G. (2014). Academic and professional discourse. En M. 
La organización retórica del género Manual de Economía: Un discurso en tránsito disciplinar / G. PARODI, E. BoudON, C. JuLIO

Lacorte (ed.), Routledge Encyclopedia of Hispanic Applied Linguistics (pp. 459476). London: Routledge.

Bondi, M. (1995). World of fact and world of hypothesis in Economics Textbooks. En V. Negri Zamagni (ed.), Annale 1994/1995, Università degli Studi di Bologna, Facoltà di Economia, Sede di Forlì (pp. 341-354). Cesena: Società Editrice "Il Ponte Vecchio".

Bondi, M. (1996a). Language variations across genres: Quantifiers and worlds of reference in (and around) economics textbooks. Asp, 11(14), 33-53.

Bondi, M. (1996b). World of fact and world of hypothesis in Economics textbooks. En V. Negri Zamagni (coord.), Annale 1994/1995, Università degli Studi di Bologna, Facoltà di Economia, Sede di Forlì (pp. 341-354); Cesena, Società Editrice "Il Ponte Vecchio".

Bondi, M. (1999). English across genres: Language variation in the discourse of Economics. Modena: Il Fiorino.

Bondi, M. (2002). Attitude and episteme in academic discourse: Adverbials of stance across genres and moves. Textus, 15(2), 249-264.

Bondi, M (2010). Arguing in economics and business discourse: Phraseological tools in research articles. Bulletin Suisse de Linguistique Appliquée, 2, 219-234.

Boudon, E. y Parodi, G. (2014). Artefactos multisemióticos y discurso académico de la Economía: construcción de conocimientos en el género Manual. Revista Signos. Estudios de Lingüistica, 47(85), 164-195.

Brown, V. (1993). Decanonizing discourse: Textual analysis and the history of economic thought. En W. Henderson, A. Dudley-Evans \& R. Blackhouse (eds.), Economics as Language (pp. 64-84). London: Routledge.

Cubo de Severino, L. (2005). Los manuales universitarios. En L. Cubo de Severino (coord.), Los textos de la Ciencia. Principales clases de discurso académicocientifico (pp. 325-335). Córdoba: Comunicarte.

Dimopoulos, K., Koulaidis, V. \& Sklaveniti, S. (2005). Towards a framework of socio-linguistic analysis of science textbooks: The Greek case. Research in Science Education, 35, 173-195.

Dudley-Evans, T. \& Henderson, W. (1990.) The language of economics: The discourse. London: Modern English Publications/British Council.

Erreygers, G. \& Jacobs, G. (eds.). (2005). Language, Communication and the Economy. Amsterdam/Philadelphia: John Benjamins.

Flowerdew, J. (2002). Introduction: Approaches to analysis of academic discourse in English. En J. Flowerdew (ed.), Academic Discourse (pp. 1-17). London: Longman.

Goldschmidt, N. \& Szmrecsanyi, B. (2007). What do economists talk about? A linguistic analysis of published writing in economic journals. American Journal of Economics and Sociology, 66(2), 335-378. 
Hall, B. y López, M. (2011). Discurso Académico: Manuales universitarios y prácticas pedagógicas. Literatura y Lingüistica, 23, 167-192.

Henderson, W. (1982). Metaphor in economics. Economics, 18(4), 147-157.

Henderson, W., Dudley-Evans, A. \& Backhouse, R. (eds.). (1993). Economics as language. London: Routledge.

Hewings, A. (1990). Aspects of the language of Economics Textbooks. En A. Dudley-Evans \& W. Henderson (eds.), The language of Economics: The analysis of Economics discourse (pp. 29-42). London: Modern English Publications/ British Council.

Hyland, K. (1998). Hedging in scientific research articles. Amsterdam: John Benjamins.

Hyon, S. (1996). Genre in three traditions: Implications for ESL. TESOL Quarterly, 30, 693-722.

Ibáñez, R. (2008). El Texto Disciplinar y el acceso al conocimiento desde el análisis del género: ¿Regulación del conocimiento o persuasión? En Parodi, G. (ed.), Géneros académicos y géneros profesionales: Accesos discursivos para saber y hacer (pp. 219-245). Valparaíso: EUV.

Kanoksilapatham, B. (2007). Rhetorical moves in biochemistry research articles. En D. Biber, U. Connor \& T. Upton (eds.), Discourse on the move (pp. 73119). Amsterdam: John Benjamins.

Kearsey, J. \& Turner, S. (1999) Evaluating textbooks: the role of genre analysis. Research in Science and Technological Education, 17(1), 35-43.

Klamer, A. (1990) The textbook presentation of economic discourse. En W. Samuels (ed.), Economics as discourse. An analysis of the language of Economics (pp. 129-154). London: Kluwer.

Kress, G. (2010). Multimodality. A social semiotic approach to contemporary communication. Londres: Routledge.

Kuhn, T. (1970). The structure of scientific revolutions. Chicago: University of Chicago Press.

Maci, S. \& Salas, M. (eds.). (2012). Genre variation in academic communication. Emerging disciplinary trends. Bérgamo, Italia: CELSB Libreria Universitaria.

Malavasi, D. (2012). Research articles in Business and Marketing: A comparative analysis of English Discussions and Italian Conclusioni. En S. Maci \& M. Salas (eds.), Genre variation in academic communication. Emerging disciplinary trends (pp. 21-44). Bérgamo, Italia: CELSB Librería Universitaria.

Martin, J.; Maton, K. \& Matruglio, E. (2010). Historical cosmologies: Epistemology and axiology in Australian secondary school history discourse. Revista Signos. Estudios de Lingüistica, 43(74), 433-463.

Martínez, J. (2012). Descripción y variación retórico-funcional del género Tesis Doctoral: Un análisis desde dos disciplinas y dos comunidades disciplinares 
a partir del corpus Te DICE-2010. Tesis Doctoral, Universidad Católica de Valparaíso, Valparaíso, Chile.

Martínez, J. M. (2009). El léxico del español de los negocios. Investigaciones Lingüisticas en el Siglo XXI, 169-187.

Mateo, J. (2007). El lenguaje de las ciencias económicas. En E. Alcaraz, J. Mateo, \& F. Yus (eds.), Las lenguas profesionales y académicas (pp.191-203). Barcelona: Ariel.

McCloskey, D. (1983). The rhetoric of Economics. Journal of Economic Literature, 31(2), 482-504.

McCloskey, D. (1985). The rhetoric of Economics. Madison: University of Wisconsin Press.

McCloskey, D. (1994). Knowledge and persuasion in Economics. Cambridge: CUP.

McCloskey, D. (1999). Economical writing. USA: Waveland.

Mosini, V. (2011). Reassessing the paradigm of Economics: Bringing positive Economics back into the normative framework. London: Routledge.

Moyano, E. (2012). Argumentación en Economía: Negociación de una interpretación. RILL, 17(1-2), 1-12.

Moyano, E. (ed.) (2013). Aprender Ciencias y Humanidades: Una cuestión de lectura y escritura. Buenos Aires: Universidad Nacional de General Sarmiento.

Nevado, A. (2012). La enseñanza del lenguaje especifico de la Economía. Disponible en www.facli.unibo.it/NR/rdonlyres/.../navadoeikonomos1.pdf.

Oakey, D. (2005). Academic vocabulary in academic discourse: The phraseological behaviour of evaluation in Economics research articles. En E. TogniniBonelli \& G. Del Lungo (eds.), Strategies in academic discourse (pp. 169-183). Ámsterdam: John Benjamins.

Oteíza, T. y Pinto, D. (eds.). (2011). En (re)construcción: Discurso, identidad y nación en los manuales escolares de Historia y de Ciencias Sociales. Santiago: Editorial Cuarto Propio.

Parodi, G. (2008a). Géneros del discurso escrito: Hacia una concepción integral desde una perspectiva sociocognitiva. En G. Parodi (ed.), Géneros académicos y géneros profesionales: Accesos discursivos para saber y hacer (pp. 17-36). Valparaíso: EUV.

Parodi, G. (2008b). La organización retórica el género Manual: ¿¿Una colonia encadenada? En G. Parodi (ed.), Géneros académicos y géneros profesionales: Accesos discursivos para saber y hacer (pp. 169-198). Valparaíso: EUV.

Parodi, G. (2008c). El género Manual y su organización retórica en cuatro disciplinas científicas. Entre la abstracción y la concreción. En G. Parodi (ed.), Géneros académicos y géneros profesionales: Accesos discursivos para saber y hacer (pp. 199-218). Valparaíso: EUV.

Parodi, G. (Ed.) (2010a). Alfabetización académica y profesional en el Siglo XXI: Leer y escribir desde las disciplinas. Santiago de Chile: Planeta. 
Parodi, G. (2010b). Multisemiosis y Lingüística de Corpus: Artefactos (multi) semióticos en los textos de seis disciplinas en el Corpus PUCV-2010. Revista de Lingüistica Teórica y Aplicada, 48(2), 33-70.

Parodi, G. (2012). ¿Qué se lee en los estudios doctorales?: Estudio empírico basado en géneros a través del discurso académico de seis disciplinas. Revista de Lingüistica Teórica y Aplicada, 50(2), 89-119.

Parodi, G. (2014a). Traversing disciplinarity: Multisemiotic artifacts across genres in the PUCV-UCSC-2013 Academic Corpus of Spanish. Ponencia presentada en la 7a Conferencia Internacional de Multimodalidad (7-ICOM), junio 2014, Universidad Politécnica de Hong Kong, Hong Kong.

Parodi, G. (2014b). Genre organisation in specialised discourse: Disciplinary variation across university textbooks. Discourse Studies, 16(1), 65-87.

Parodi, G. \& Bocca, R. (2013). Academic discourse of Economics: From discourse genre and multisemiotic artifact definitions until the design of a learner corpus. Ponencia presentada en el 2nd Colloquium Linguistics in the Asia-Pacific. PUCV, Viña del Mar, Chile. 14 de octubre.

Parodi, G. y Burdiles, G. (2013). El pronombre 'ello' como mecanismo encapsulador en el discurso académico de la Economía: Función gramatical y referencia textual. Ponencia presentada en el XX Congreso Internacional de la Sociedad Chilena de Lingüística, Universidad Católica de la Santísima Concepción, Concepción.

Parodi, G.; Venegas, R.; Ibáñez, R. y Gutiérrez, R. (2008). Los géneros del discurso en el Corpus PUCV-2006: Criterios, definiciones y ejemplos. En G. Parodi (ed.), Géneros académicos y géneros profesionales: Accesos discursivos para saber y hacer (pp. 43-59). Valparaíso: EUV.

Royce, T. (1999). Visual-verbal intersemiotic complementarity in the Economist magazine. Tesis doctoral, The University of Reading, Reading, Inglaterra.

Russell, D.; Lea, M.; Parker, J.; Street, B. \& Donahue, T. (2009). Exploring notions of genre in "Academic Literacies" and "Writing across the curriculum": Approaches across countries and contexts. En Ch. Bazerman, A. Bonini \& D. Figueiredo (eds.), Genre in a changing world (pp. 395-423). Indiana: Parlor Press.

Samuels, W. (ed.) (1990). Economics as discourse: An analysis of the language of Economists. Amsterdam: Kluwer.

Stagnaro, D. (2010). Expresión de la evaluación en conclusiones de artículos del dominio de la economía. En Actas del Congreso Regional de la Cátedra UNESCO en Lectura y Escritura: "Cultura Escrita y Politicas Pedagógicas en las Sociedades Latinoamericanas Actuales”. Cátedra UNESCO - Universidad Nacional de General Sarmiento. Argentina.

Stagnaro, D. (2011). ¿Qué analizan los economistas?: Exploración del proceso “analizar" en artículos de Desarrollo Económico del período 2000-2010. Ponen- 
cia presentada en el VII Congreso Internacional de la Asociación de Lingüística Sistémico-Funcional de América Latina. Universidad Nacional del Litoral, Santa Fe, Argentina.

Stagnaro, D. (2012). Negociación interpersonal en las conclusiones del artículo de investigación de economía: exploración de los recursos expresivos del compromiso frente a las propuestas. Ponencia presentada en el XIII Congreso de la Sociedad Argentina de Lingüística. San Luis, Argentina.

Swales, J. (1981). Aspects of Article Introductions. Birmingham: University of Aston.

Swales, J. (1990). Genre analysis. English in academic and research settings. Cambridge: Cambridge University Press.

Swales, J. (1993). The paradox of value: Six treatments in search of the reader. En W. Henderson, T. Dudley-Evans \& R., Backhouse (eds.), Economics and language (pp. 223-239). London: Routledge.

Swales, J. (1995) The role of Textbooks in AEP writing research. English for Specific Purposes, 14(1), 3-18.

Swales, J. (2004). Research genres: Explorations and applications. Cambridge: Cambridge University Press.

Swales, J. (2009). Worlds of genre-metaphors of genre. En Ch. Bazerman, A. Bonini \& D. Figueiredo (eds.), Genre in a changing world (pp. 3-16). Indiana: Parlor Press.

Van Leeuwen, T. (2011). The language of colour. An introduction. New York: Routledge.

Venegas, R. (2014). El trabajo final de Grado de Licenciatura: Caracterización discursiva y andamiaje didáctico. Conferencia presentada en el 3er Congreso Nacional de Pedagogía Universidad de Los Lagos, Osorno, Chile. 